\title{
Directed forgetting of visual symbols: Evidence for nonverbal selective rehearsal
}

\author{
Kathleen L. Hourihan \\ University of Illinois at Urbana-Champaign, Champaign, Illinois
}

AND

Jason D. Ozubko and Colin M. MacLeod

University of Waterloo, Waterloo, Ontario, Canada

\begin{abstract}
Is selective rehearsal possible for nonverbal information? Two experiments addressed this question using the item method directed forgetting paradigm, where the advantage of remember items over forget items is ascribed to selective rehearsal favoring the remember items. In both experiments, difficult-to-name abstract symbols were presented for study, followed by a recognition test. Directed forgetting effects were evident for these symbols, regardless of whether they were or were not spontaneously named. Critically, a directed forgetting effect was observed for unnamed symbols even when the symbols were studied under verbal suppression to prevent verbal rehearsal. This pattern indicates that a form of nonverbal rehearsal can be used strategically (i.e., selectively) to enhance memory, even when verbal rehearsal is not possible.
\end{abstract}

One of the best-known findings in the memory literature is that pictures are remembered much better than words (e.g., Shepard, 1967). This remarkable capacity to remember pictures is well demonstrated in Standing, Conezio, and Haber's (1970) study in which subjects were presented with 1,560 different photographs of scenes to study in 2-h sessions over a 4-day period. When their memory was tested on a subset of the studied pictures, using a two-alternative forced choice recognition test, the subjects were approximately $90 \%$ accurate in their recognition of the studied pictures, whether tested in their original orientation or in reverse orientation. Moreover, this picture superiority effect in memory holds not only for complex pictures such as those in Standing et al., but also for simple object line drawings (e.g., Durso \& O’Sullivan, 1983).

Despite the obviously superior memory for pictures over words, it is unclear whether pictorial material can benefit from a rehearsal process in the same manner as verbal material. Shaffer and Shiffrin (1972) observed that, unlike word memory, picture memory did not benefit from additional poststimulus rehearsal time. They concluded that, although forms of visual rehearsal might be possible in certain contexts, rehearsal was not applied to complex visual information such as pictures. However, subsequent research was unable to replicate their results, and Proctor (1983) determined that picture memory did benefit from additional poststimulus rehearsal time, except under the particular circumstances used by Shaffer and Shiffrin.

Shaffer and Shiffrin (1972) presented pictures for varying durations and also varied the poststimulus rehearsal period in a randomized, rather than blocked, manner. Varying both factors makes it difficult to predict the time available to encode each item. Proctor (1983) concluded that subjects likely gave up on any form of visual rehearsal when they realized that the presentation scheme was highly unpredictable. His data support the claim that both random duration and random poststimulus intervals are necessary for picture memory not to improve with additional rehearsal time. When only one of these factors was randomized and the other was blocked, he found that picture recognition did improve with increased poststimulus rehearsal time. Proctor concluded that rehearsal of pictures is possible, although not routine.

Watkins (1985) disagreed with Proctor's (1983) view that pictorial rehearsal is used only under predictable circumstances and pointed out that Proctor's data could also be explained by subjects' using a more flexible rehearsal strategy. That is, Watkins proposed that subjects can rehearse pictures other than the one most recently presented (a form of distributed rehearsal), thus eliminating any differences in memory based on poststimulus intervals. Proctor (1985), however, questioned the strength of the evidence for this claim. In particular, he cited low statistical power in his own experiment (Proctor, 1983) that Watkins had interpreted as good evidence for distributed rehearsal of pictorial materials. Proctor (1985) generally concluded that pictorial rehearsal was unlikely to be as flexible as Watkins claimed.

Watkins and colleagues (Graefe \& Watkins, 1980; Watkins \& Graefe, 1981; Watkins, Peynircioğlu, \& Brems,

K. L.Hourihan, hourihan@illinois.edu 
1984) have provided several good pieces of evidence supporting the argument that pictures can be readily rehearsed. Watkins and Graefe showed that subjects can selectively rehearse one picture out of a set of three, four, or even eight pictures (line drawings). Using a procedure similar to that in Graefe and Watkins, pictures were presented in a series, one after another, with one of them cued for rehearsal following presentation of the series. As was observed previously (Graefe \& Watkins, 1980), Watkins and Graefe found that, later, cued pictures were recognized better than uncued pictures. The question arises, however, whether such memory benefits for pictures derive from a truly nonverbal form of rehearsal, or whether verbal rehearsal contributes to the improved recognition. Certainly, for photographs, subjects could have chosen to verbally rehearse components of the pictures that they were able to label. The primary goal of the present study was to examine whether nonverbal rehearsal is possible, whether it can be done selectively, and whether it can contribute to improved memory in a manner similar to verbal rehearsal.

Watkins and Graefe (1981) began each picture series by presenting the names of the pictures that would be shown in the series; moreover, to cue the to-be-rehearsed picture, that picture's name was presented following the picture series. Thus, it is likely that verbal rehearsal of the cued picture's name contributed to the benefit in memory. Rehearsal of the picture's verbal label exclusively could not have created the memory benefit, however, because the distractor items on the recognition test included different views of the same nominal picture. Simply rehearsing the label "chair," for example, could not have allowed subjects to selectively recognize the particular view of the chair presented in the series and then cued for rehearsal, so some form of visual processing likely took place during the rehearsal interval.

Further supporting the possibility of a truly nonverbal form of rehearsal, Watkins et al. (1984) specifically instructed subjects either to rehearse a picture's verbal label or to rehearse the picture visually (i.e., the subjects were told to "maintain an image of the picture ... and to scan the image continuously with the mind's eye"; Watkins et al., 1984, p. 555). Subjects were shown a set of line drawings, each accompanied by its name. The next picture followed either immediately or after a 15 -sec blank interval, in which the subjects were asked to rehearse the item just presented either verbally or visually. Half of the studied pictures (both rehearsed and unrehearsed) were tested on a picture fragment task (visual), and half on a word fragment task (verbal). Test performance was better for rehearsed than for unrehearsed pictures only when the form of test matched the form of rehearsal. Given the very specific transfer in performance from type of rehearsal to type of test, Watkins et al. concluded that this was good evidence that pictures can be rehearsed nonverbally.

Clearly, however, not all forms of pictures can be rehearsed nonverbally (i.e., in the absence of verbal labels). Wright et al. (1990) compared the effects of poststimulus rehearsal time for travel scenes and kaleidoscope pictures. Whereas recognition of the travel scenes improved with increased rehearsal time, recognition of the kaleidoscope pictures did not. Wright et al. postulated that subjects could not rehearse the kaleidoscope pictures because it was too difficult to assign a verbal label or a description to them, unlike the travel scenes. Wright et al.'s experiments showed some evidence for rehearsal of pictures but also showed that naming picture stimuli contributes substantially to rehearsal effects.

Thus, there is evidence in the literature that pictures can be rehearsed and that this results in improved memory for the rehearsed pictures, relative to the unrehearsed pictures (Graefe \& Watkins, 1980; Proctor, 1983; Watkins \& Graefe, 1981; Wright et al., 1990). There is even evidence suggesting that rehearsal may be able to take a nonverbal form (Watkins et al., 1984). However, whereas Watkins and Graefe showed that a picture can be selected for rehearsal out of a set of recently presented pictures, no study thus far has provided convincing evidence that pictorial rehearsal can be performed as strategically as verbal rehearsal in the manner proposed by Watkins (1985). That is, in all of the studies described, rehearsal effects have been examined by showing that pictures followed by a blank period are remembered better than those not followed by a blank period, when subjects are under instructions to remember all of the pictures. Consequently, evidence is lacking that pictures can truly be selected for rehearsal in a strategic manner in the absence of verbal labels, as Watkins proposed was possible.

The goal of the present study was, therefore, to determine whether pictorial rehearsal can be performed and whether it can be performed in a strategic manner, similar to verbal rehearsal (e.g., Rundus, 1971). That is, can subjects choose to selectively rehearse pictures (or not) in response to experimenter instructions? Rather than examining rehearsal of pictures by manipulating poststimulus rehearsal intervals, we presented pictorial materials in a paradigm that has robustly shown strategic rehearsal effects with verbal materials: the item method directed forgetting paradigm (see MacLeod, 1998, for a review). In this paradigm, subjects typically are given a list of words to study. Each word is followed by an individual instruction either to remember or to forget that word, usually under a global instruction that forget words will not be tested. When memory is then tested for all the studied items regardless of memory instruction, a directed forgetting effect is observed: More remember words than forget words are retrieved, whether measured by recall or by recognition.

The item method directed forgetting effect has long been ascribed to selective rehearsal (e.g., Basden, Basden, \& Gargano, 1993; MacLeod, 1998). Because subjects are aware that each word will be followed by a memory instruction and that this instruction might be to forget the word, they hold rehearsal in abeyance until the memory instruction is received. Remember words are subsequently rehearsed, whereas forget words are discarded before they can be entered into the rehearsal set. As a result, this situation is ideal for testing whether pictorial material can be rehearsed strategically, selecting only some items for nonverbal rehearsal. Because duration is constant for remember and forget trials, the additional rehearsal af- 
forded to remember items likely takes place cumulatively, distributing rehearsals across the study list (cf. Hourihan $\&$ Taylor, 2006). If pictures cannot be rehearsed flexibly in this manner and can be rehearsed (or not) only immediately following presentation, observation of a directed forgetting effect would be unlikely, assuming that the next presented picture immediately follows the previous trial's instruction.

Few studies have examined pictorial materials in the context of item method directed forgetting. Basden and Basden (1996) and Goernert, Widner, and Otani (2007) found directed forgetting effects on free recall of the names of studied pictures, but they used readily nameable line drawing stimuli, all from a single category (animals). More recently, Quinlan, Taylor, and Fawcett (in press) systematically compared item method directed forgetting of pictures and words (the verbal label or name of each picture). In their experiment, subjects studied either pictures or words and were tested with either pictures or words, resulting in four groups. For both of the groups in which pictures were presented at study, a directed forgetting effect was evident at test (regardless of whether words or pictures were presented at test). The magnitude of the directed forgetting effect was substantially reduced for studied pictures, relative to studied words, but the effect was present in all conditions. That is, even though pictures are highly memorable and nonverbal, they are still subject to directed forgetting effects. Hauswald and Kissler (2008) found a similar, small-magnitude directed forgetting effect, using more complex photographs.

However, as Quinlan et al. (in press) pointed out in their discussion, the pictures that they used-like those in Basden and Basden (1996) - were highly nameable (indeed, the pictures were highly nameable by design, to permit testing of studied pictures using words, and vice versa). Therefore, it is entirely possible that their observed directed forgetting effects were due to subjects' naming the pictures at the time of study and then selectively rehearsing those verbal labels. Although Hauswald and Kissler (2008) argued that their photographs were not readily labeled, they did not measure or record any actual naming behavior, nor did they prevent any verbal rehearsal from occurring. Thus, as with most of the studies of pictorial rehearsal (Graefe \& Watkins, 1980; Watkins \& Graefe, 1981; Wright et al., 1990), it is not clear whether the observed rehearsal effects were truly due to pictorial rehearsal or whether rehearsal of verbal labels (either presented or generated at study) contributed strongly to the observed effects. Therefore, the present study was conducted to determine whether pictures can be rehearsed selectively and strategically in the absence of verbal rehearsal of picture names.

In our first experiment, we examined whether selective rehearsal of pictures was possible when the pictures were not easily named. Instead of the commonly used line drawings of objects, we used distinctive patterns and shapes derived by modifying characters from the Wingdings font (see the Appendix). The symbols were easily distinguished from each other but did not appear to have an obvious or "correct" name or label. However, in pilot testing of these symbols, many subjects anecdotally reported that they could and did name approximately half of the symbols. We therefore included a final phase following recognition to systematically record which symbols the subjects had named during study, so that we could subsequently compare recognition of named versus unnamed symbols. Thus, in the first experiment, we examined how selective rehearsal differed for symbols that were spontaneously named at study (allowing verbal rehearsal of those symbol names) versus symbols that were not named at study (and therefore, were unlikely to be verbally rehearsed).

In our second experiment, our goal was to prevent symbol naming and, hence, verbal rehearsal by including a verbal suppression task at the time of study. Occupying verbal processing with a secondary task should be sufficient to prevent any verbal form of selective rehearsal from occurring during the study phase (cf. Richardson $\&$ Baddeley, 1975). Therefore, if selective rehearsal were still possible (as would be evidenced by occurrence of a directed forgetting effect), it likely would be possible only in a nonverbal form. Thus, the goal of the second experiment was to provide stronger evidence regarding whether nonverbal selective rehearsal of symbols would take place under conditions that prevented verbal rehearsal.

\section{EXPERIMENT 1}

The goal of Experiment 1 was to determine whether selective rehearsal is possible for pictures that are unlikely to be named. The item method directed forgetting paradigm provides a suitable testing ground, given the strong evidence that the directed forgetting effect observed with this paradigm derives from selective rehearsal (see MacLeod, 1998, for a review). We know that directed forgetting effects can be produced by selective verbal rehearsal. Can directed forgetting effects also be produced by selective nonverbal rehearsal?

We began by pilot testing our materials. This pretesting revealed that subjects reported naming approximately half of the symbols during study, perhaps an indication of the pervasive nature of verbal processing. Further testing revealed that performance for the named symbols approached ceiling, so a $24-\mathrm{h}$ retention interval was included in Experiment 1 to reduce overall performance. We took advantage of the fact that half of the symbols were named and half were not named to make a direct comparison between the two subsets.

The subjects were given a list of these symbols to study, half followed by a remember instruction and half followed by a forget instruction. All the symbols were later tested by yes-no recognition. We sought to determine whether only named symbols were subject to directed forgetting, via selective verbal rehearsal of those names, or whether even symbols that were not named could display a directed forgetting effect, via selective nonverbal rehearsal of the symbols themselves. To test this, after the recognition test, the subjects completed an additional task in which they were asked to indicate for each symbol whether they had given that symbol a name or label during the study phase. They were further asked to name every symbol, providing 
the name that they had thought of during study or generating a name for the first time if they had not named a particular symbol at study.

If the previously reported directed forgetting effects for pictures were driven purely by selective rehearsal of those pictures' verbal labels, and if selective nonverbal rehearsal of the symbols is not possible, unnamed symbols should not be subject to directed forgetting effects. Only the symbols given names that could be rehearsed verbally should produce a directed forgetting effect. If, however, nonverbal selective rehearsal is possible for unnamed symbols, a directed forgetting effect should still be evident.

\section{Method}

Subjects. Twenty-four undergraduate students from the University of Waterloo participated in exchange for course credit or payment.

Materials and Apparatus. The 96 stimuli were created by modifying characters from the Wingdings font to create novel symbols that were readily differentiated from each other (see the Appendix). Study lists were created from a random selection of 64 symbols on a per-subject basis. The remaining symbols not presented at study were reserved to serve as distractors on the recognition test. All the stimuli were presented in black on a white background.

The stimuli were presented using a Pentium IV computer with a 17-in. CRT monitor, using E-Prime software (v.1.1.4.1) to randomize presentation order. Memory instructions consisted of a row of capital letters ("RRRRR" for remember and "FFFFF" for forget) and were presented in 18-point black Courier font.

Procedure. The subjects were informed that they would be given a list of symbols to study for a later memory test, but the nature of the test was left unspecified. The study phase presented 64 symbols, 32 followed by a remember instruction and 32 followed by a forget instruction. The subjects were told that they should try to remember symbols followed by a remember instruction but that symbols followed by a forget instruction did not need to be remembered, because they would not be tested. Symbol order and memory instruction were randomly determined for each subject. Each study trial began with a fixation cross ("+") presented at the center of the screen for $500 \mathrm{msec}$. Following a 500 -msec blank screen, the symbol was presented at the center of the screen for $2,000 \mathrm{msec}$. A blank screen was then presented for $500 \mathrm{msec}$, followed by the memory instruction ("RRRRR" or "FFFFF") at the center of the screen for 2,000 msec. A 500-msec blank screen preceded the next trial.

Following completion of the study phase, the subjects were dismissed from the lab; they returned the following day for the memory test, resulting in a retention interval of $18-30 \mathrm{~h}$. The mean retention interval was $24.3 \mathrm{~h}(S D=2.78)$; retention interval was not a significant covariate in any later analyses of recognition performance. Upon returning to the lab, the subjects were given instructions for the recognition test. They were asked to respond yes to all symbols that they had seen before, regardless of the memory instruction shown after the symbol during study. All of the studied symbols (32 remember and 32 forget) were presented, along with the remaining 32 symbols not presented at study. Presentation order was randomly determined for each subject. On each test trial, the symbol was presented at the center of the screen and remained visible until the subject responded yes by pressing the " $\mathrm{m}$ " key or no by pressing the " $\mathrm{z}$ " key. A 500-msec blank preceded the next trial.

After completing the recognition test, the subjects took part in the symbol-naming phase. For each of the 64 studied symbols, they were asked whether they had given it a name or label when they had first studied it. The subjects responded yes by pressing the "Y" key or no by pressing the "N" key. They were then asked to provide the name that they had given the symbol (if they had named it during study) or to create a name for the symbol (if they had not named it during study). The subjects responded by typing the name.

\section{Results and Discussion}

The recognition results are shown in the top half of Table 1 . In the critical analysis, we conditionalized the subjects' hit rates on whether they had spontaneously named symbols during the study phase. As can be seen in the top half of Table 2, the subjects named about half of the studied symbols, approximately the same proportion as were spontaneously named in Wright et al.'s (1990) experiment using kaleidoscope pictures. As is indicated by the low variance of these proportions, naming behavior was consistent across individuals. Furthermore, their responses indicated that they had named the same proportions of remember and forget symbols $[t(23)=0.48, p=.63]$.

The conditionalized hit rates were analyzed in a 2 (remember vs. forget instruction) $\times 2$ (named vs. not named) within-subjects ANOVA. ${ }^{1}$ A significant directed forgetting effect was observed overall $\left[F(1,22)=5.73, M S_{\mathrm{e}}=\right.$ $\left.0.02, p<.05, \eta_{\mathrm{p}}^{2}=.21\right]$. In addition, named symbols were recognized better than unnamed symbols $[F(1,22)=4.89$, $\left.M S_{\mathrm{e}}=0.05, p<.05, \eta_{\mathrm{p}}^{2}=.18\right]$, but no significant interaction was observed $[F(1,22)<1]$.

We further examined whether the relative ease of naming individual symbols had affected their later recognition. On the basis of the naming behavior that the subjects reported for each symbol, we collapsed across subjects and performed a median split on how frequently a particular symbol was named at study, resulting in two sets

Table 1

Experiments 1 and 2: Mean Proportions (and Standard Errors) for Symbols Correctly Recognized As a Function of Memory Instruction and Whether Symbols Were Spontaneously Named at Study

\begin{tabular}{|c|c|c|c|c|c|c|}
\hline \multirow[b]{2}{*}{ Instruction } & \multicolumn{2}{|c|}{ Overall } & \multicolumn{2}{|c|}{$\begin{array}{c}\text { Named at } \\
\text { Study }\end{array}$} & \multicolumn{2}{|c|}{$\begin{array}{c}\text { Not Named } \\
\text { at Study }\end{array}$} \\
\hline & $M$ & $S E$ & $M$ & $S E$ & $M$ & $S E$ \\
\hline \multicolumn{7}{|c|}{ Experiment 1} \\
\hline Remember & .72 & .03 & .75 & .04 & .65 & .04 \\
\hline Forget & .60 & .03 & .69 & .04 & .56 & .04 \\
\hline \multicolumn{7}{|c|}{ Experiment 2} \\
\hline Remember & .69 & .02 & .75 & .04 & .66 & .03 \\
\hline Forget & .60 & .03 & .73 & .04 & .58 & .03 \\
\hline
\end{tabular}

Note-False alarm rates were $.19(.04)$ and $.19(.03)$ in Experiments 1 and 2 , respectively.

Table 2

Experiments 1 and 2: Mean Proportions (and Standard Errors) for Symbols Spontaneously Named at Study, As a Function of Memory Instruction

\begin{tabular}{cccccc}
\hline & \multicolumn{2}{c}{$\begin{array}{c}\text { Proportion } \\
\text { Named }\end{array}$} & & \multicolumn{2}{c}{$\begin{array}{c}\text { Proportion } \\
\text { Not Named }\end{array}$} \\
\cline { 2 - 3 } \cline { 5 - 6 } Instruction & $M$ & $S E$ & & $M$ & $S E$ \\
\hline Experiment 1 & & \\
Remember & .26 & .02 & & .23 & .02 \\
Forget & .25 & .02 & & .26 & .02 \\
Overall & .50 & .04 & & .50 & .04 \\
& \multicolumn{2}{c}{ Experiment 2} & & \\
Remember & .16 & .02 & & .35 & .02 \\
Forget & .16 & .02 & & .33 & .02 \\
Overall & .32 & .04 & .68 & .04 \\
\hline
\end{tabular}


of items: easy-to-name items and difficult-to-name items. On a per-subject level of analysis, actual naming behavior reflected this item difference: The subjects named more of the easy-to-name items $(M=.69, S D=.13)$ than of the difficult-to-name items $(M=.35, S D=.11)[t(94)=$ 14.27, $p<.01]$. Recognition performance was then reanalyzed in a 2 (easy- vs. difficult-to-name) $\times 2$ (remember vs. forget instruction) $\times 2$ (named vs. not named) repeated measures ANOVA. ${ }^{2}$ This analysis revealed a significant directed forgetting effect $\left[F(1,19)=4.66, M S_{\mathrm{e}}=\right.$ $\left.0.05, p<.05, \eta_{\mathrm{p}}^{2}=.20\right]$. The main effect of naming was also significant $\left[F(1,19)=3.05, M S_{\mathrm{e}}=0.11, p<.01\right.$, $\left.\eta_{\mathrm{p}}^{2}=.59\right]$. There was no main effect of ease of naming, nor were any of the interactions significant (all $p \mathrm{~s}>.22$ ). Therefore, the directed forgetting effects observed were not driven by the relative ease of naming the individual symbols: Both easy-to-name and difficult-to-name symbols produced equivalent directed forgetting effects.

Surprisingly, then, although naming a symbol certainly improved memory for that symbol, it had no effect on whether that symbol could be intentionally forgotten. That is, the magnitude of the observed directed forgetting effect was equivalent for named and unnamed symbols. If only verbal rehearsal could be performed in a strategic manner, only the named symbols would have been selectively rehearsed, and therefore, the unnamed symbols would not have shown a directed forgetting effect. This clearly was not the case.

We see three possible explanations for why there was a directed forgetting effect for both named and unnamed symbols. First, it may be that "unnamed" symbols were simply symbols that were more difficult to name or that had less consistent names, so that the subjects were more likely to forget those names by the time they were tested. The "named" symbols, on the other hand, were better recognized because re-presentation of those symbols reminded the subjects of the names that they had generated at study, and this additional information enhanced recognition. However, the analysis of the individual items' ease of naming produced null effects, so this explanation seems unlikely. Second, it may be that a form of nonverbal selective rehearsal was implemented by the subjects for all the studied symbols. The reason for the superior recognition of named symbols would therefore be that these symbols benefitted from both nonverbal rehearsal and verbal rehearsal of the generated symbol names, a kind of dual-coding advantage (see Paivio, 1969) that fits with the pattern observed by Wright et al. (1990). Third, the subjects may have verbally rehearsed the named symbols and nonverbally rehearsed the unnamed symbols. Verbal rehearsal may have resulted in better encoding than did nonverbal rehearsal, thereby explaining the superior recognition of named symbols.

\section{EXPERIMENT 2}

In Experiment 2, our goal was to explore whether the symbols that subjects reported as "unnamed" in Experiment 1 truly were unnamed and, thus, must have been selectively rehearsed in a nonverbal manner to produce the observed directed forgetting effect. To ascertain whether nonverbal selective rehearsal is possible, symbol naming must be prevented from occurring in the first place. To prevent verbal rehearsal during the study phase, we selected a simple verbal suppression task modeled after that used by Guérard and Tremblay (2008, Experiment 2). This suppression task simply requires subjects to repeat letters aloud at a fixed pace and was shown to selectively interfere with a verbal task (relative to a spatial task) in Guérard and Tremblay's results.

To the extent that the directed forgetting effect is driven purely by verbal rehearsal, eliminating the possibility of verbal rehearsal should eliminate the effect. Certainly, the proportion of symbols that subjects report naming at the time of presentation should be reduced - if not eliminated - relative to Experiment 1. If being able to rehearse verbally is necessary to selectively rehearse symbols and, thus, to produce a directed forgetting effect, occupying verbal processes via verbal suppression should reduce or eliminate the effect. However, if nonverbal selective rehearsal is possible, a directed forgetting effect should still be observed. If, as in Experiment 1, some symbols are still named despite the verbal suppression task, their rehearsal should be prevented or impaired, thus eliminating a directed forgetting effect for named items by preventing selective rehearsal.

\section{Method}

Subjects. Twenty-nine different individuals from the same pool took part.

Materials and Apparatus. The materials and apparatus were identical to those in Experiment 1, with the addition of single letters (A, B, C, and D) presented at fixation as part of the verbal suppression task. The letters were printed in the same font as the memory instructions. They appeared in black during the practice phase and in gray during the study phase.

Procedure. The study phase was similar to that in Experiment 1 , but with the addition of the verbal suppression task. Prior to the study phase, the subjects completed a practice phase, to familiarize them with the pacing of the suppression task. In the practice phase, the letters A, B, C, and D were presented sequentially at fixation at a rate of 1 letter per $500 \mathrm{msec}$ (with no pauses between letters). The subjects were asked to say each displayed letter aloud as soon as they saw it on the screen. The displayed letter cycled, in order, five times (i.e., 20 letters) and was then followed by 4 cycles in which half of the letters were not displayed and, instead, blank screens were shown. The subjects were instructed to continue saying all 4 letters, in order, even during the blank screens on which nothing was displayed. This was to encourage the subjects to keep pace with the letter repetition even while the letters were not actually on the screen, as would be the case during the study phase. Following 2 cycles of full display, an additional 29 cycles of letters were presented, with decreasing numbers of letters actually displayed as the cycles went on, for a total of 40 cycles of practice (i.e., 160 letters). This practice phase lasted $80 \mathrm{sec}$.

After the practice phase, the study phase began. Each trial began with one cycle of letter presentation where the letters were presented slightly above fixation and displayed in gray. A symbol was then presented at the center of the screen for 2,000 msec. The symbol was removed for $500 \mathrm{msec}$, during which time the letter A was displayed to remind the subjects of the pacing of the verbal suppression task. The memory instruction was then displayed at the center of the screen for $1,500 \mathrm{msec}$. The next trial began immediately after (i.e., with one cycle of letter presentation). Following completion of the study phase, the test phase began and was identical to that in Experi- 
ment 1 (including completion of the symbol-naming task following the recognition test). It was not necessary to include a retention interval, as in Experiment 1, because the added difficulty of the verbal suppression task during study ensured nonceiling performance.

\section{Results and Discussion}

The results are shown in the bottom half of Table 1. Although an overall directed forgetting effect was observed $[t(28)=4.64, p<.001]$, as in Experiment 1, our emphasis was on hit rates conditionalized on whether the subjects reported having named symbols during study. Importantly, as can be seen in the bottom half of Table 2, the subjects named far fewer symbols in Experiment 2 than in Experiment $1[t(51)=3.37, p<.01]$, indicating that the addition of the verbal suppression task served its purpose: to minimize naming and, thus, to reduce or eliminate verbal rehearsal of presented symbols. In addition, in the present experiment, there was no difference in the proportions of remember versus forget symbols that were named $[t(28)=0.65, p=.52]$, again indicating that any directed forgetting effects observed could not be due to differential naming of remember and forget symbols.

As in Experiment 1, recognition performance was analyzed in a 2 (remember vs. forget instruction) $\times 2$ (named vs. not named) within-subjects ANOVA. A significant directed forgetting effect was observed overall $[F(1,24)=$ $10.64, M S_{\mathrm{e}}=0.01, p<.05, \eta_{\mathrm{p}}^{2}=.31$ ], replicating Experiment 1 . In addition, named symbols were recognized better than unnamed symbols $\left[F(1,24)=25.66, M S_{\mathrm{e}}=\right.$ $\left.0.06, p<.05, \eta_{\mathrm{p}}^{2}=.52\right]$. Although no significant interaction was observed ( $p>.10)$, interpreting the interaction raises two issues. First, unlike in Experiment 1, the proportions of items that were named versus not named were unequal, with many more items unnamed. Thus, analyzing both named and not named items with a single interaction term is not ideal, because named means will be less reliable than unnamed means. Second, some subjects did not name any symbols. Although this is good in terms of the goal of the experiment, the data from these subjects could not be included in our ANOVA, because these subjects had missing data points for the named cells. Given these issues with the interaction term, and because the focus of Experiment 2 was on whether unnamed symbols can be selectively rehearsed, the named and unnamed symbols were analyzed separately in paired-sample $t$ tests. These tests revealed no directed forgetting effect for named symbols $[t(24)=0.76$, $p=.46]$ but did reveal a significant directed forgetting effect for unnamed symbols $[t(28)=3.52, p<.01] .^{3}$

Finally, we examined whether the relative ease of naming individual symbols had affected later recognition of those symbols. As in Experiment 1, we performed a median split on how frequently a particular symbol was named across subjects; once again, easy-to-name items $(M=.45, S D=.12)$ were actually named more often than difficult-to-name items $(M=.18, S D=.08)[t(94)=$ $12.85, p<.01]$. Recognition performance was then reanalyzed in a 2 (easy- vs. difficult-to-name) $\times 2$ (remember vs. forget instruction) $\times 2$ (named vs. not named) repeated measures ANOVA. ${ }^{4}$ This analysis revealed a significant directed forgetting effect $\left[F(1,18)=17.86, M S_{\mathrm{e}}=0.02\right.$, $\left.p<.01, \eta_{\mathrm{p}}^{2}=.50\right]$. The main effect of naming was also significant $\left[F(1,18)=36.15, M S_{\mathrm{e}}=0.11, p<.01, \eta_{\mathrm{p}}^{2}=\right.$ .67]. Finally, there was a significant three-way interaction $\left[F(1,18)=6.94, M S_{\mathrm{e}}=0.03, p<.05, \eta_{\mathrm{p}}^{2}=.28\right]$. This three-way interaction was driven by the fact that for named symbols, there was no directed forgetting effect for easy-to-name symbols but there was a directed forgetting effect for difficult-to-name symbols. ${ }^{5}$ There was no main effect of ease of naming, nor were any of the other interactions significant (all $p \mathrm{~s}>.15$ ). Therefore, the directed forgetting effect observed for unnamed symbols was not driven by the relative ease of naming the individual symbols; both easy- and difficult-to-name symbols produced equivalent directed forgetting effects when unnamed.

The results support the idea that nonverbal selective rehearsal can occur in the context of item method directed forgetting. First, consider that the verbal suppression task prevented — or at least minimized — symbol naming and verbal rehearsal. Although some symbols were still spontaneously named by the subjects and named symbols did benefit relative to unnamed symbols, in recognition [collapsing across memory instruction: named, $M=.74$, $S E=.04$, vs. unnamed, $M=.60, S E=.03 ; t(24)=3.26$, $p<.01$ ], significantly fewer symbols were named in Experiment 2 than in Experiment 1. Moreover, the named symbols did not show a directed forgetting effect, indicating that they were not selectively rehearsed throughout the study phase, because verbal rehearsal was prevented by the verbal suppression task (we will return to this point later). Second, and most important, the unnamed symbols did produce a directed forgetting effect. This is a quite striking dissociation. The directed forgetting effect for the unnamed symbols occurred despite selective verbal rehearsal's likely not being possible because of the verbal suppression task. Yet the only reason to expect better recognition of remember than of forget symbols would be that remember symbols were rehearsed more than forget symbols - the standard interpretation of item method directed forgetting (see MacLeod, 1998). Thus, by inference, because verbal rehearsal was not possible for unnamed symbols yet a directed forgetting effect was observed for them, those symbols must have been selectively rehearsed in a nonverbal manner.

Put simply, the simultaneous verbal suppression task during encoding prevented the selective verbal rehearsal of named pictures. As a consequence, and because item method directed forgetting hinges on rehearsal, there was no directed forgetting effect for named pictures. However, the verbal suppression task should not have prevented nonverbal selective rehearsal from being applied to unnamed pictures. Consequently, because there was a directed forgetting effect for unnamed pictures, we conclude that selective rehearsal had to have occurred and that it had to have been nonverbal.

\section{GENERAL DISCUSSION}

The goal of the present experiments was to determine whether nonverbal rehearsal can operate in a strategic, selective manner, similar to verbal rehearsal. To test this, 
we used the item method directed forgetting paradigm, which has, for decades, been viewed as revealing selective rehearsal processes (MacLeod, 1975, 1998). We therefore saw this paradigm as an ideal one for determining whether pictures can be selectively rehearsed when rehearsal of verbal labels is not possible. In using this paradigm, we have made two key observations across our experiments. First, we have shown that nonverbal rehearsal is, in fact, possible. Second, in response to our principal question, we have provided evidence that strategic, nonverbal selective rehearsal is possible, although we have also shown that verbal rehearsal may be preferred when it is possible. We have also demonstrated that spontaneously generating a verbal label - a name - for a pictorial item at the time of encoding produces a substantial benefit in later memory, independently of whether selective verbal rehearsal of the generated name is possible.

Evidence for nonverbal selective rehearsal was strongest in Experiment 2, in which verbal rehearsal was prevented by the concurrent performance of a verbal suppression task during the study phase. Experiment 1 also provided evidence for nonverbal rehearsal, in that the unnamed symbols produced a directed forgetting effect whose magnitude was the same as that for the named symbols. However, in Experiment 1, a directed forgetting effect was also observed for the named symbols, which likely was due to selective verbal rehearsal of the remember symbol names throughout the study phase. This might be interpreted as indicating that the unnamed symbols had also been named (because there was nothing to prevent naming from occurring) but that their names were less memorable and, thus, the subjects reported not having named those symbols when tested $24 \mathrm{~h}$ later. In Experiment 2, however, verbal selective rehearsal was made considerably less likely because of the verbal suppression task, and thus the directed forgetting effect seen only for the unnamed symbols (and not for the named symbols) was due to a form of selective nonverbal rehearsal.

The finding that nonverbal selective rehearsal occurs fits well with Proctor's (1983) finding that picture recognition does benefit from additional rehearsal time when study conditions are predictable. It is also important to note, however, that verbal rehearsal seems to have been the preferred strategy, at least in our experiments. In our first experiment, when given no explicit instructions otherwise, the subjects spontaneously named about half of the presented symbols (like the subjects in Wright et al., 1990) and selectively rehearsed the remember symbols to produce a directed forgetting effect. It appears that once a visual stimulus is named, the strong preference thereafter is to verbally rehearse the name, rather than to nonverbally rehearse the visual stimulus. It may be possible to reveal nonverbal rehearsal only when verbal rehearsal is prevented.

Even when verbal processing was made difficult due to a concurrent verbal task (in Experiment 2), the subjects still spontaneously named approximately a third of the presented symbols, despite not being able to selectively rehearse those names. Although subjects generally favor verbal processing, which is rather unsurprising, given the predominantly verbal nature of rehearsal in general (e.g., Rundus, 1971), nonverbal rehearsal is possible (e.g., Watkins et al., 1984). As well, our experiments show for the first time that nonverbal rehearsal can be implemented in a controlled, selective manner to produce directed forgetting effects.

Evidence of cumulative, distributed rehearsal in directed forgetting has been provided with verbal materials (Hourihan \& Taylor, 2006), in that recall and recognition of forget words improves with increased rehearsal time; remember words do not show this effect (but see Woodward, Bjork, \& Jongeward, 1973). This indicates that remember words are rehearsed at times other than immediately following their presentation; otherwise, words with longer rehearsal intervals should have shown improved memory. Because we did not manipulate rehearsal time, we do not have direct evidence that a similar form of distributed but nonverbal rehearsal took place in our experiments, or that remember symbols were rehearsed nonverbally only in the period immediately following the memory instruction. However, we speculate that nonverbal distributed rehearsal of pictures is a distinct possibility (cf. Watkins, 1985).

An additional principal finding in our experiments is that generating a symbol name produced a substantial memory benefit for those symbols when tested later. Even in Experiment 2 , in which symbol naming was difficult because of a concurrent verbal task that prevented verbal rehearsal, the relatively fewer symbols that were named showed a substantial memory benefit, relative to the unnamed symbols. Thus, even when symbols cannot be further rehearsed verbally, the act of having generated a symbol name at the time of presentation improves encoding sufficiently that recognition of an unrehearsed, named symbol is still better than recognition of a nonverbally rehearsed, unnamed symbol, as was seen in Experiment 2. This fits neatly with a dual-coding framework (Paivio, 1969).

The memory benefit obtained from generating an item name at the time of encoding is not surprising, given the robustness of the generation effect (e.g., Slamecka \& Graf, 1978) and the production effect (MacLeod, Gopie, Hourihan, Neary, \& Ozubko, in press) in memory for verbal materials. When MacLeod and Daniels (2000) examined the generation effect in the context of item method directed forgetting, they found that the directed forgetting effect was eliminated for items generated at study, although it was present for words that were simply read. More recently, Hourihan and MacLeod (2008) found that produced items - words read aloud at study - did not show directed forgetting effects either, although words read silently did.

One key difference between the nature of generation in the present study and that in the typical generation and production manipulations is that there was no correct answer to be generated in our study. In the MacLeod and Daniels (2000) experiment, subjects were required to generate a particular target word in response to a definition by retrieving that item from semantic memory. In 
the Hourihan and MacLeod (2008) experiment, subjects were required to correctly read the presented word. In our experiment, subjects could generate any response they chose and were, therefore, not seeking to retrieve the correct solution from semantic memory or to produce a word's correct pronunciation. Thus, the name generation process added additional contextual information that served to benefit later recognition but still allowed selective rehearsal to improve memory for the remember items over the forget items. Retrieval from semantic memory, not simply improving memory performance per se, may be the key to eliminating the directed forgetting effect for generated items, a possibility that we intend to pursue elsewhere.

Throughout this article, we have treated verbal rehearsal and nonverbal rehearsal almost as if they were independent processes. However, this is not truly our intention: We are not claiming that verbal and nonverbal rehearsal are independent, nonoverlapping processes. We have good reason to believe that no form of verbal rehearsal took place for any of the symbols in our unnamed conditions: Subjects reported not providing a verbal name for the symbols, and, in Experiment 2, verbal rehearsal would have been difficult, if not impossible. However, it is entirely possible that nonverbal processes were combined with verbal processes for any of the named symbols in our experiments, because we did nothing to prevent this from occurring, nor could we measure whether rehearsal of named symbols was purely verbal. In fact, we think it likely that at least some nonverbal rehearsal was afforded all study symbols and that named symbols were afforded additional verbal rehearsal in conditions in which this was possible.

The lack of a directed forgetting effect for named symbols in Experiment 2, however, might seem to speak against this idea that verbal and nonverbal rehearsal coexist: If no verbal rehearsal could take place due to the verbal suppression task, why were the named symbols not selectively rehearsed nonverbally, which would then have produced a directed forgetting effect anyway? Although we cannot answer this for certain, we can speculate that something akin to the verbal overshadowing effect (e.g., Schooler \& Engstler-Schooler, 1990) may have been in play. That is, whereas the named symbols had the potential to be selectively rehearsed nonverbally, the fact that they had been spontaneously named precluded that form of rehearsal because of the strong association formed between the symbol and its generated name. Once a picture is named, the only rehearsal option that may be considered is verbal rehearsal, which here was precluded by the verbal suppression task.

The goal of the present project was not to separate and define the individual influences of verbal and nonverbal rehearsal processes, but simply to determine whether selective rehearsal of pictorial materials is possible in a nonverbal form, particularly when verbal rehearsal is not possible. We believe that we have shown in these experiments that selective nonverbal rehearsal is possible and that it can be revealed under the right circumstances.
Thus, although the directed forgetting effects in past studies in which pictures have been used (Basden \& Basden, 1996; Hauswald \& Kissler, 2008; Quinlan et al., in press) may well have been driven by verbal rehearsal of the picture names, it is entirely possible that nonverbal selective rehearsal also contributed to those effects. Moreover, whenever stimuli that are not readily named are used, nonverbal rehearsal of these stimuli is possible, and that nonverbal rehearsal can be deployed selectively, just as is the case for verbal rehearsal. Our results therefore side more with Watkins's (1985) view that pictorial rehearsal can operate in a flexible manner, rather than being abandoned under unpredictable circumstances (Proctor, 1983, 1985). Verbal labels allow and may even promote verbal rehearsal of pictures, but these verbal labels are not essential for rehearsal to occur. As we have demonstrated, nonverbal rehearsal is also possible and can be selectively engaged to improve memory.

\section{AUTHOR NOTE}

This research was supported by Natural Sciences and Engineering Research Council (NSERC) of Canada Discovery Grant A7459 and by NSERC PGS-D and CGS-D scholarships. We thank Samantha Chaves, Grace Hsiao, and Brandon Vasquez for their assistance in data collection. Correspondence concerning this article should be addressed to K. L. Hourihan, Department of Psychology, University of Illinois at UrbanaChampaign, 838 Psychology Building, 603 East Daniel Street, Champaign, IL 61820 (e-mail: hourihan@illinois.edu).

\section{REFERENCES}

Basden, B. H., \& Basden, D. R. (1996). Directed forgetting: Further comparisons of the item and list methods. Memory, 4, 633-653. doi:10.1080/741941000

Basden, B. H., Basden, D. R., \& Gargano, G. J. (1993). Directed forgetting in implicit and explicit memory tests: A comparison of methods. Journal of Experimental Psychology: Learning, Memory, \& Cognition, 19, 603-616. doi:10.1037/0278-7393.19.3.603

Durso, F. T., \& O'Sullivan, C. S. (1983). Naming and remembering proper and common nouns and pictures. Journal of Experimental Psychology: Learning, Memory, \& Cognition, 9, 497-510. doi:10.1037/0278-7393.9.3.497

Goernert, P. N., Widner, R. L., JR., \& Otani, H. (2007). Classification accuracy across multiple tests following item method directed forgetting. Quarterly Journal of Experimental Psychology, 60, 1178-1186.

Graefe, T. M., \& Watkins, M. J. (1980). Picture rehearsal: An effect of selectively attending to pictures no longer in view. Journal of Experimental Psychology: Learning, Memory, \& Cognition, 6, 156-162. doi:10.1037/0278-7393.6.2.156

GuÉrard, K., \& TREmblay, S. (2008). Revisiting evidence for modularity and functional equivalence across verbal and spatial domains in memory. Journal of Experimental Psychology: Learning, Memory, \& Cognition, 34, 556-569. doi:10.1037/0278-7393.34.3.556

Hauswald, A., \& Kissler, J. (2008). Directed forgetting of complex pictures in an item method paradigm. Memory, 16, 797-809.

Hourihan, K. L., \& MacLEOD, C. M. (2008). Directed forgetting meets the production effect: Distinctive processing is resistant to intentional forgetting. Canadian Journal of Experimental Psychology, 62, 242246. doi:10.1037/1196-1961.62.4.242

Hourihan, K. L., \& TAYLOR, T. L. (2006). Cease remembering: Control processes in directed forgetting. Journal of Experimental Psychology: Human Perception \& Performance, 32, 1354-1365. doi:10.1037/0096 $-1523.32 .6 .1354$

MacLEOD, C. M. (1975). Long-term recognition and recall following directed forgetting. Journal of Experimental Psychology: Human Learning \& Memory, 1, 271-279. doi:10.1037/0278-7393.1.3.271

MacLeod, C. M. (1998). Directed forgetting. In J. M. Golding \& C. M. 
MacLeod (Eds.), Intentional forgetting: Interdisciplinary approaches (pp. 1- 57). Mahwah, NJ: Erlbaum.

MacLeod, C. M., \& Daniels, K. A. (2000). Direct versus indirect tests of memory: Directed forgetting meets the generation effect. Psychonomic Bulletin \& Review, 7, 354-359.

MacLeod, C. M., Gopie, N., Hourihan, K. L., Neary, K. R., \& Ozubкo, J. D. (in press). The production effect: Delineation of a phenomenon. Journal of Experimental Psychology: Learning, Memory, \& Cognition.

PAIVIO, A. (1969). Mental imagery in associative learning and memory. Psychological Review, 76, 241-263.

PROCTOR, R. W. (1983). Recognition memory for pictures as a function of poststimulus interval: An empirical clarification of existing literature. Journal of Experimental Psychology: Learning, Memory, \& Cognition, 9, 256-262. doi:10.1037/0278-7393.9.2.256

Proctor, R. W. (1985). How flexible is picture rehearsal? Discussion of Watkins' comment. Journal of Experimental Psychology: Learning, Memory, \& Cognition, 11, 825-828. doi:10.1037/0278-7393 $.11 .1-4.825$

Quinlan, C. K., Taylor, T. L., \& Fawcett, J. M. (in press). Directed forgetting: Comparing pictures and words. Canadian Journal of Experimental Psychology.

RichaRdson, J. T. E., \& BAdDELEY, A. D. (1975). The effect of articulatory suppression in free recall. Journal of Verbal Learning \& Verbal Behavior, 14, 623-629. doi:10.1016/S0022-5371(75)80049-1

Rundus, D. (1971). Analysis of rehearsal processes in free recall. Journal of Experimental Psychology, 89, 63-77. doi:10.1037/h0031185

SCHOOLER, J. W., \& ENGSTLER-SCHOOLER, T. Y. (1990). Verbal overshadowing of visual memories: Some things are better left unsaid. Cognitive Psychology, 22, 36-71. doi:10.1016/0010-0285(90)90003-M

Shaffer, W., \& Shiffrin, R. M. (1972). Rehearsal and storage of visual information. Journal of Experimental Psychology, 92, 292-296. doi:10.1037/h0032076

SHEPARD, R. N. (1967). Recognition memory for words, sentences, and pictures. Journal of Verbal Learning \& Verbal Behavior, 6, 156-163. doi:10.1016/S0022-5371(67)80067-7

Slamecka, N. J., \& Graf, P. (1978). The generation effect: Delineation of a phenomenon. Journal of Experimental Psychology: Human Learning \& Memory, 4, 592-604. doi:10.1037/0278-7393.4.6.592

Standing, L., Conezio, J., \& Haber, R. N. (1970). Perception and memory for pictures: Single-trial learning of 2500 visual stimuli. Psychonomic Science, 19, 73-74.
WATKINS, M. J. (1985). Strategies of picture rehearsal: A comment on Proctor's (1983) article. Journal of Experimental Psychology: Learning, Memory, \& Cognition, 11, 821-824. doi:10.1037/0278-7393.11.1-4.821

Watkins, M. J., \& Graefe, T. M. (1981). Delayed rehearsal of pictures. Journal of Verbal Learning \& Verbal Behavior, 20, 276-288. doi:10.1016/S0022-5371(81)90428-X

Watkins, M. J., PeynircioğLu, Z. F., \& Brems, D. J. (1984). Pictorial rehearsal. Memory \& Cognition, 12, 553-557.

WoodWARD, A. E., JR., BJork, R. A., \& JongeWARD, R. H., JR. (1973). Recall and recognition as a function of primary rehearsal. Journal of Verbal Learning \& Verbal Behavior, 12, 608-617. doi:10.1016/S0022 -5371(73)80040-4

Wright, A. A., Cook, R. G., Rivera, J. J., Shyan, M. R., Neiworth, J. J., \& Jitsumori, M. (1990). Naming, rehearsal, and interstimulus interval effects in memory processing. Journal of Experimental Psychology: Learning, Memory, \& Cognition, 16, 1043-1059. doi:10.1037/0278-7393.16.6.1043

\section{NOTES}

1. One subject's data could not be analyzed because that subject did not name any forget symbols, resulting in no data for that cell for that subject.

2. Four subjects were missing data in one or more of the cells required for this analysis (i.e., they did not name any of the symbols in a particular cell), so these subjects were not included in this analysis.

3. The degrees of freedom reported for the $t$ tests here are not the same, because 4 subjects did not name any remember symbols, leaving no data to compute for these subjects.

4. Ten subjects were missing data in one or more of the cells required for this analysis (i.e., they did not name any of the symbols in a particular cell), so these subjects were not included in this analysis.

5. One caveat concerning interpreting this interaction is that the data of only 19 of the 29 subjects in Experiment 2 could be included in this ANOVA, because 10 subjects did not name any symbols in one or more of the six cells. Moreover, the small proportion of difficult-to-name items that were actually named means that the observed directed forgetting effect is based on a comparison of approximately three remember with three forget items (approximately $9 \%$ of the total items presented). Thus, we do not feel that it would be wise to draw strong theoretical conclusions from a three-way interaction that is limited to only two thirds of all the subjects and less than $10 \%$ of all the items. 
APPENDIX

Stimuli Used in Experiments 1 and 2

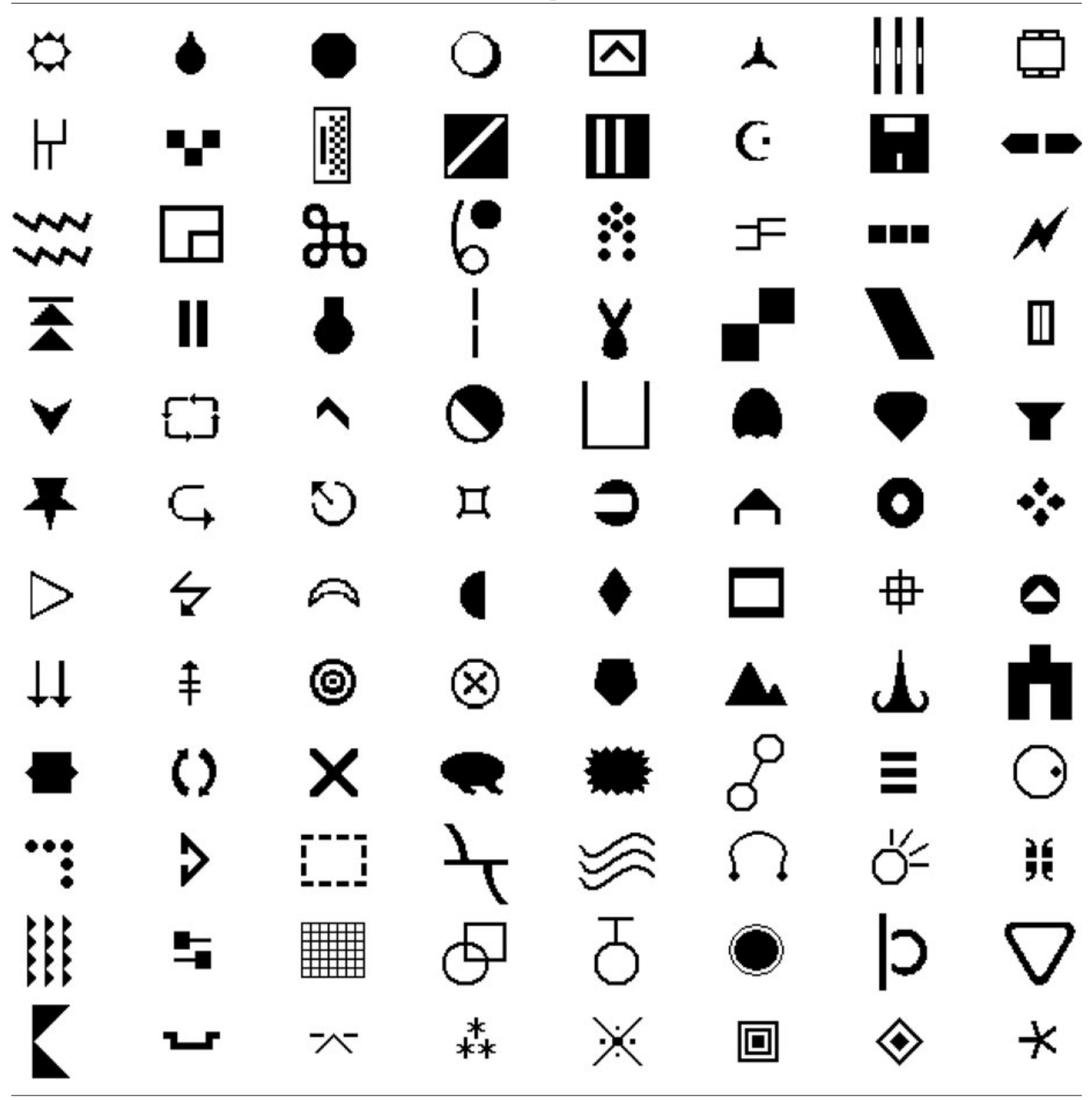

(Manuscript received December 4, 2008;

revision accepted for publication May 28, 2009.) 